\title{
CIRCULATION PATTERNS AND SHORELINE RESPONSE INDUCED BY SUBMERGED BREAKWATERS
}

\author{
Mara Villani ${ }^{1}$, Judith Bosboom ${ }^{2}$, Marcel Zijlema ${ }^{2}$, Marcel J.F. Stive ${ }^{2}$ \\ Submerged shore-parallel breakwaters for coastal defence (henceforth SBWs) are a good compromise between the \\ need to mitigate the effects of waves on the coast and the ambition to ensure the preservation of the landscape and \\ water quality. However, if not properly designed, such structures can force circulation patterns that enhance shoreline \\ erosion rather than shoreline accretion. Numerical models can be used to investigate the structure-induced circulation \\ patterns and the resulting shoreline response. However, being computationally demanding these models are most \\ suitable for advanced stages of the design process. The aim of this paper is to present a simple criterion to \\ qualitatively identify whether an accretive or erosive circulation pattern can be expected in the lee of the structures. \\ The criterion is based on analytical considerations and builds on the model presented by Bellotti (2004, 2007). It is \\ validated against the non-hydrostatic free surface numerical model SWASH (Zijlema et al. 2011) and experiments \\ performed by Haller et al. (2002). The validation indicates that the proposed analytical model is capable of providing \\ a rapid first assessment of the potential shoreline response mode for SBW design.
}

Keywords: coastal defence; submerged breakwaters; coastal currents; design criterion

\section{INTRODUCTION}

Due to their low visual and environmental impact submerged structures - having a crest level constantly below the sea surface - are an appealing solution for coastal defence. However, if not properly designed, submerged shore-parallel breakwaters (SBWs) may cause shoreline erosion rather than shoreline accretion as a result of structure-induced circulation currents. In a review of the shoreline response to submerged structures Ranasinghe and Turner (2006) pointed out that 7 out of 10 considered cases have resulted in unexpected shoreline erosion.

The need to fully understand the hydraulic behaviour of SBWs has triggered a large amount of research into this topic. Nowadays, various wave-resolving and wave-averaged numerical models are available to investigate nearshore hydrodynamic and morphodynamic phenomena induced by submerged structures. Being computationally demanding these models are generally more compatible with an advanced stage of the design process. On the other hand, simplified models based on analytical considerations and empirical formulas can offer rapid preliminary predictions with satisfactory accuracy.

A simple response-function model for the case of a single SBW was proposed by Ranasinghe et al. (2010). They presented a functional relationship linking environmental and geometrical properties of the system to the shoreline mode of response, i.e. accretive or erosive. In doing so they used the type of structure-induced circulation current as a proxy for the shoreline mode of response (Ranasinghe et al. (2006); an erosional 2-cell circulation system is formed by onshore wave-induced currents over the structure that diverge in the entire lee zone and return off-shore again at the gaps transporting sediments out of the protected area. In turn, for an accretive 4-cell circulation system, an additional converging flow closer to the shoreline due set-up gradients promotes sediment deposition. Vicinanza et al. (2009) proposed a simplified model to predict the main currents induced by low-crested structures in the protected area. The model combines a converging flow near the shoreline with an onshore flow over the structure that is due to overtopping only. No account is taken of a nonzero onshore flow over the breakwater forced by wave breaking on a submerged structure and the storage of water behind submerged structures. Using a simple analytical model Bellotti $(2004,2007)$ investigates the water level set-up and currents induced by wave breaking over the crest of discontinuous submerged barriers. A similar model is proposed by Zanuttigh (2008), which explicitly represents filtration across and undertow over the structure. By expressing the functional dependency between the water level set-up in the lee of the barrier to the net inshore discharge over the barrier crest these models describe the hydrodynamics around SBWs in analogy with the operation of a pump plant. Neither Bellotti (2007) nor Zanuttigh (2008) solve for set-up driven flow near the shoreline or suggest a particular relationship with the possible shoreline mode of response.

In the present work, a simplified analytical model is presented to qualitatively identify the circulation patterns in the case of waves normally incident to a series of shore-parallel submerged structures for coastal defence. Following Ranasinghe et al. (2006), we assume that the type of 2DH

\footnotetext{
${ }^{1}$ Dipartimento di Ingegneria Civile e Ambientale (DICEA), University of Rome La Sapienza, via Eudossiana 18, Rome, 00184, Italy

${ }^{2}$ Faculty of Civil Engineering and Geosciences, Delft University of Technology, Delft, The Netherlands
} 
circulation pattern (2-cell or 4-cell) can be used as a proxy for the shoreline mode of response. In doing so, the present work provides SBW designers of a tool to make a rapid first assessment of the potential shoreline response (erosion or accretion) in a preliminary stage of the design process.

The model is validated against a non-hydrostatic numerical model for free-surface rotational flows (Zijlema et al. 2011) that has been successfully verified for a range of experimental data. This model is an open source tool developed at Delft University of Technology and has been successfully verified for a range of experimental data. We present comparisons between the here presented simple model, the numerical model and a set of data from laboratory experiments performed by Haller et al. (2002) on a fixed barred beach with periodically spaced rip channels. In the validations, we focus on the mean water level variations along two selected transects and the type of circulation currents.

The paper is organized as follows: in Section 2 the driving forces of the accretive and erosive circulation patterns and the equations of the model are described; Section 3 describes the validation of the results and in Section 4 the conclusions and the suggestions for further improvements are presented.

\section{CONCEPTUAL MODEL}

The present simple model is aimed at identifying the mode of response of the shoreline to discontinuous SBWs via a critical analysis of the structure-induced variations in mean water levels as a result of normally incident waves. These set-up variations govern the flow patterns in the protected area behind the structures (see Figs.1-2).

The onshore forces due to wave breaking over the structure are balanced by a combination of onshore flow and set-up over the breakwater depending on the level of lateral confinement. The lateral confinement can be expressed as the ratio between the length $L_{b}$ of the structures and the length $L_{g}$ of the gaps (Fig.4). The set-up or piling up $\eta_{2}$ at the inshore toe of the breakwater (Fig.3) has its maximum for total lateral confinement and decreases as the gap length increases. Mass conservation requires that the water flowing onshore over the structure returns off-shore again through the gaps. We assume that the natural surf zone width is smaller than the distance from the SBW to the shoreline and that mean water level variations at the gap due to set-down can be neglected. Hence, the head determining the velocity of the outflow at the gaps is equal to $\eta_{2}$.

The incoming waves directly approaching the shore through the gaps steepen and finally break due to water depth limitations: the related wave set-up at the shoreline is referred to as $\eta_{g}$. The wave set-up related to the breaking of the re-shoaled transmitted waves behind the structure is referred to as $\eta_{b}$. The reduction of wave energy due to the wave breaking over the structure causes the transmitted waves to break closer to the shoreline than those at the gaps and with a smaller wave set-up.

For a small enough distance between the shoreline and the structure, we can assume the mean water level behind the structures to be raised with a constant amount in the cross-shore direction equal to the maximum structure-induced wave set-up, $\eta_{2}$. Therefore, close to the shoreline in the lee of the structures the total wave set-up is supposed to be the sum of the set-up due to the confinement of the system, $\eta_{2}$, and the set-up due to the breaking of the transmitted waves, $\eta_{b}$.

The above makes clear that the series of shore-parallel SBWs partially acts like a dam because of geometry reasons (limited distance between the shoreline and the structure and length of the gaps small in comparison with the structure length); the storage of water forced in the sheltered area by wave breaking over the SBW is dependent on the degree of confinement of the system with a maximum for total lateral confinement (no gap between the structures).

As mentioned before, the inflow of water over the barrier must leave the system again at the gaps by means of diverging currents in the lee of the structures. The resulting circulation system in the lee of the breakwater is composed of two symmetric circulation cells (Fig.1). Close to the shoreline, depending on the direction of the alongshore gradient in mean water level, the alongshore flow direction may reverse and be directed towards the centreline of the barrier. This is the case if the total wave set-up at the gaps $\left(\eta_{g}\right)$ is greater than behind the structures $\left(\eta_{2}+\eta_{b}\right)$ driving converging currents near the shoreline. This leads to a circulation system composed of four circulation cells (Fig.2). The type of 2DH circulation pattern (2-cell or 4-cell) is now used as a proxy for the shoreline mode of response; the diverging currents in a 2-cell system drive sediments out of the protected area causing shoreline erosion, whereas the converging currents in a 4-cell system cause shoreline accretion. 


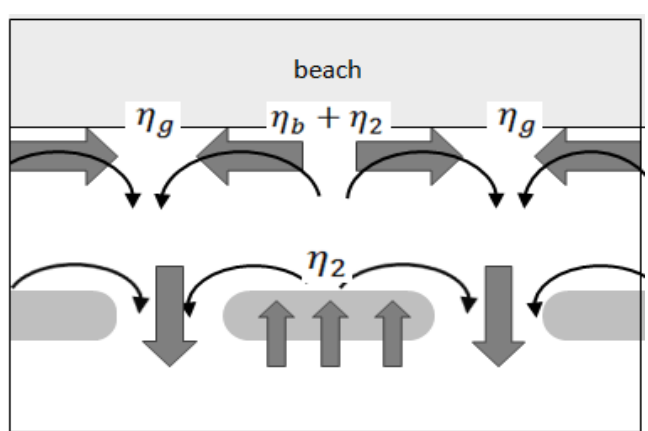

Fig. 1 - Erosive 2-cell circulation pattern

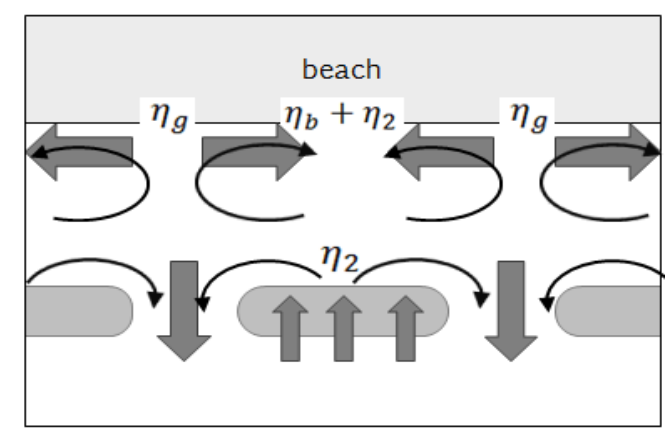

Fig. 2 - Accretive 4-cell circulation pattern

Hence, we propose the use of the ratio $r$ (Eq.1) as a criterion to delineate between the 4-cell accretive pattern and the 2-cell erosive pattern. Accretion is expected for $r<1$ and erosion for $r>1$.

$$
r=\frac{\eta_{2}+\eta_{b}}{\eta_{g}}
$$

By applying simplified mass and momentum balances, here this criterion is formulated in terms of incident wave conditions, beach slope and SBW geometrical properties. An inherent fundamental assumption in our approach is that the alongshore variations in set-up, driving both the convergent and divergent flow, can be estimated by using the 1D cross-shore momentum balance along transects located at the middle of the gaps and at the middle of the barriers for which the maximum set-up $\eta_{2}$ is expected. The steady, depth averaged 1D momentum equation for a cross-shore transect is given by:

$$
\frac{d}{d x} \frac{q^{2}}{(h+\eta)}=-g(h+\eta) \frac{d \eta}{d x}-\frac{1}{\rho} \frac{d S_{x x}}{d x}-\frac{\tau_{b}}{\rho}
$$

where $q=u h$ is the discharge, $h$ is the still water depth, $S_{x x}$ is the radiation stress in the cross-shore $x$ direction and $\tau_{b}$ is the bottom shear stress.

The continuity equation can be written as follows (Eq.3):

$$
\frac{d q}{d x}=0
$$

According to the model presented by Bellotti (2004, 2007), we integrate Eqs.2 and 3 along the cross-shore section of a generic barrier (see Fig.3). The model does not depend on the particular shape of the barrier. The integrated equations are recalled below:

$$
\begin{aligned}
& u_{2}^{2}\left(h_{2}+\eta_{2}\right)-u_{1}^{2} h_{1}+\frac{g}{2}\left(h_{2}+\eta_{2}\right)^{2}-\frac{g}{2} h_{1}^{2}+S-V_{b}+\Gamma=0 \\
& u_{2}\left(h_{2}+\eta_{2}\right)-u_{1} h_{1}=0
\end{aligned}
$$

where $S$ is the resulting short-wave forcing, $V_{b}$ the reaction of the bottom and $\Gamma$ the fiction force term. The reader is referred to Bellotti (2007) for further details.

Resolving the continuity equation (Eq.5) for the velocity at the inshore toe of the structure, $u_{2}$, and inserting the resulting expression in the momentum balance equation (Eq.4) Bellotti obtains a relationship (Eq.6) between the flow discharge over the crest and set-up generated by the breaking of waves over structures, $\eta_{2}$ :

$$
u_{1}^{2}\left(\frac{h_{1}^{2}}{g\left(h_{2}+\eta_{2}\right)}-\frac{h_{1}}{g}\right)+u_{1}\left(\mu B \frac{h_{1}}{g}\right)+\eta_{2} \bar{h}+\frac{\eta_{2}^{2}}{2}+\frac{1}{8}\left(\frac{1}{2}+\frac{2 k_{2} h_{2}}{\sinh \left(2 k_{2} h_{2}\right)}\right) H_{t}^{2}-\frac{1}{8}\left(\frac{1}{2}+\frac{2 k_{1} h_{1}}{\sinh \left(2 k_{1} h_{1}\right)}\right) H_{i}^{2}=0
$$

where $H_{i}$ and $H_{t}$ are respectively the height of the incident and transmitted waves, $k_{1,2}$ the wave numbers and $\mu B$ originates from the friction term and does not depend on the unknowns. 


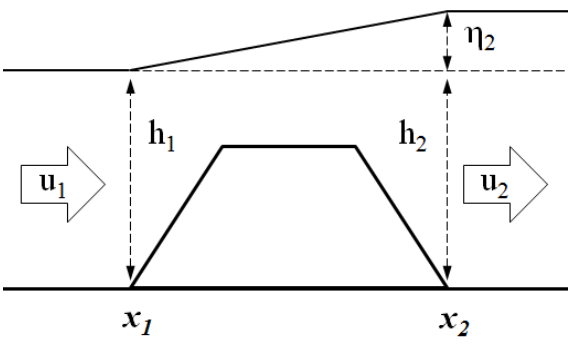

Fig. 3 - Control volume over the barrier

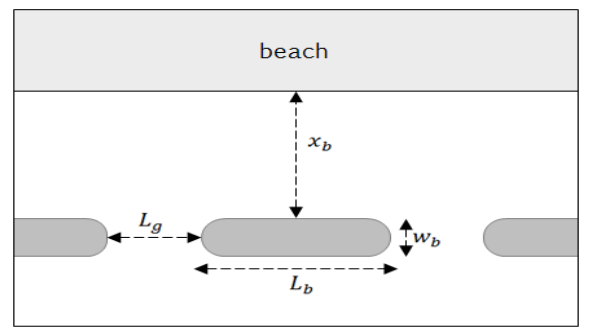

Fig. 4 - Sketch of geometrical properties in plan

Eq.6 needs to be coupled with a further equation to close the problem. As in Bellotti (2007), this extra equation is provided for by the overall continuity requirement that all the water flowing onshore over the structure must return off-shore through the gaps (Eq.7) at a velocity given by Eq. 8 that relates the hydraulic head $\eta_{2}$ to the rip current velocity $u_{g, 1}$ by means of a simple energy equation. Eqs.7 and 8 result in the closure equation (Eq. 9).

$$
\begin{aligned}
& u_{1, b} h_{1, b} L_{b}+u_{g, 1} h_{1, g} L_{g}=0 \\
& u_{g, 1}=C_{v} \sqrt{2 g \eta_{2}} \\
& u_{1, b} h_{1, b}=C_{v} \sqrt{2 g \eta_{2}} h_{1, g} \frac{L_{g}}{L_{b}}
\end{aligned}
$$

It has to be noted that Eq.9 is only one of the possible closure equations. Other physically based relationships linking the two considered flow parameters can be used. Similarly, a different method for estimating the transmitted wave height can be freely chosen.

Solving the system formed by Eqs.6 and 9 is the equivalent of finding the system operational point of a hydraulic pump where Eq.6 represents the so-called Pumping Curve and Eq.9 the System Curve. The solution is at the intersection of these two curves (Fig.6).

The idea to solve the hydrodynamics of the low-crested and submerged structures in analogy with the physical processes of a pump plant is also used by Zanuttigh et al. (2008). Their model is more comprehensive if compared to that proposed by Bellotti $(2004,2007)$ because it also takes into account the filtration through the structure and quantifies the undertow over the structure. The model proposed by Zanuttigh et al. (2008) depends on a list of 12 coefficients and parameters for which values have to be determined from literature or from calibration against experimental data, whereas Bellotti's formulation only depends on three free parameters: a friction parameter to take account of bed friction, $f_{w}$, a coefficient to represent head losses of the diverging flow, $C_{v}$, and a breaker parameter over the structure, $\gamma$. We have preferred the simplicity of the Bellotti's formulation in developing the present model without invalidating the generality of the criterion.

Here a slightly modified version of the model proposed by Bellotti (2007) is presented. In his paper Bellotti used measured values of the wave heights at the inshore and offshore toe of the barrier in computing the resulting short wave forcing term $S$ and a wave height limited by the water depth along the barrier section in computing the friction force term $\Gamma$. Since our aim is a fully predictive model, we use the deep water wave height as an initial condition and compute the transmitted wave height by means of linear wave theory and Van der Meer's empirical formula for wave transmission (Van der Meer et al. 2005). In this way the dependence of the friction term on the breaker parameter $\gamma$ can be removed assuming a linear variation over the crest of the wave height between the incident wave height at the offshore toe and transmitted wave height at the inshore toe. In doing so the wave height over the crest in Eq.9 of Bellotti (2007) is now known using the deep water wave height as input.

The next step is to compute the wave set-up in the surf zone near the shoreline by solving a reduced version of Eq.2 (Eq.10) assuming a balance between gradients in radiation stress and water level. The values of the wave set-up at the shoreline are given by Eq.11 where the subscripts $g$ and $b$ refer respectively to the section at gap and over the barrier.

$$
\frac{d \eta}{d x}=-\frac{1}{\rho g(h+\eta)} \frac{d S_{x x}}{d x}
$$




$$
\eta_{b, g}=\frac{1}{1+\frac{8}{3 \gamma_{b, g}^{2}}} h_{b \_b, g}
$$

where $h_{b}$ is the depth at breaking and is computed applying linear wave theory. The wave height to water depth ratio, $\gamma$, is assumed constant but two different values are used for the transect at the middle of the gap, $\gamma_{g}$, and the transect over the structure, $\gamma_{b}$, to take into account the presence of the rip currents (Eq.12). The effect of the opposing rip currents is to steepen the incoming waves, such that wave breaking will occur for lower values of the breaker parameter $\gamma$, see Haller and Özkan-Haller (2002). Hence:

$$
\gamma_{g}<\gamma_{b}
$$

\section{VALIDATION}

The proposed criterion (Eq.1) is here applied to four of the test cases performed by Haller et al. (2002), Table 1. Those tests represent a fixed, barred beach with rip channels and the results can be interpreted by analogy to get information about hydrodynamics at SBWs. The wave basin used to perform experiments is illustrated in Fig.5. For the present study only those test cases with orthogonal approach to the bar (B, C, D and G) have been selected.
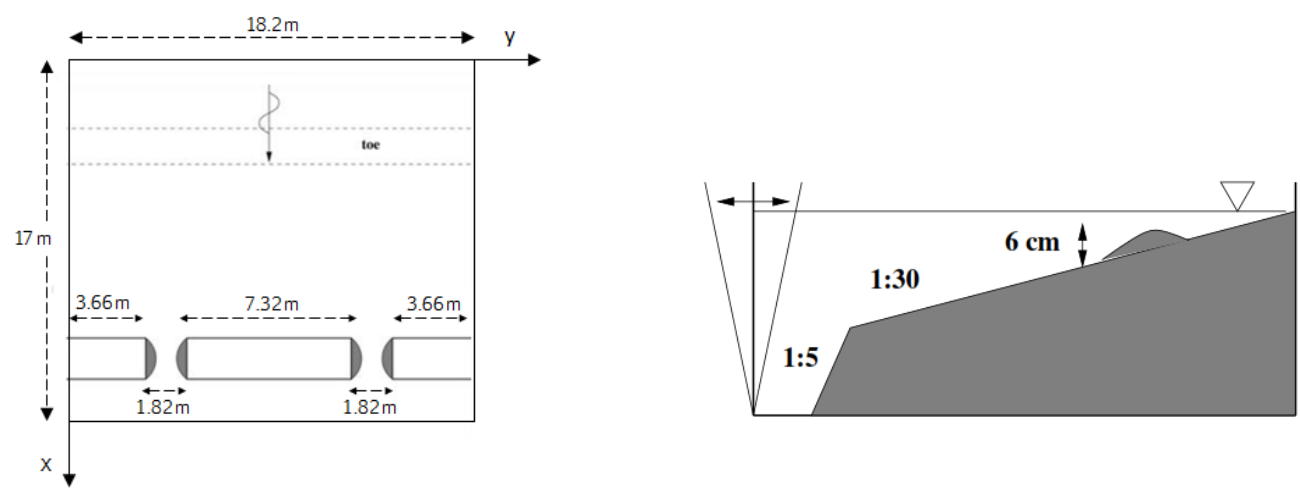

\begin{tabular}{|c|c|c|c|c|c|c|}
\hline TEST & $\mathrm{H}(\mathrm{cm})$ & $\mathrm{T}(\mathrm{sec})$ & $\theta$ (deg) & $\mathrm{h}_{\mathrm{c}}(\mathrm{cm})$ & $\mathrm{x}_{\mathrm{swl}}(\mathrm{m})$ & $\mathrm{U}_{\mathrm{r}}(\mathrm{m} / \mathrm{s})$ \\
\hline$B$ & 4.41 & 1 & 0 & 4.73 & 14.9 & 0.14 \\
\hline $\mathrm{C}$ & 4.94 & 1 & 0 & 2.67 & 14.3 & 0.18 \\
\hline $\mathrm{D}$ & 7.56 & 1 & 0 & 2.67 & 14.3 & 0.3 \\
\hline$G$ & 6.79 & 1 & 0 & 6.72 & 15.4 & 0.15 \\
\hline
\end{tabular}

Fig. 5 - Cross-section and plan view of the wave basin

The friction coefficient $f_{w}$ is set to 0.02 [-] as suggested by Haller et al. (2002) and the coefficient $C_{v}$ to 0.54 [-]. The latter is the optimum value for the Haller experiments as found by Bellotti (2004) using measured values of the flow parameters in Eq.9. Fig.6 shows the comparison between the analytical and experimental values for the 'operative points'. The empirical formula (Van der Meer at al. 2005) used to estimate the height of the transmitted waves returns values with errors in the order of magnitude of 8 to $9.5 \%$ of the measured values (Fig.7 and Table 2). The model showed to be very sensitive to this parameter so the agreement in Fig.6 can be improved by a better estimation for this parameter. 

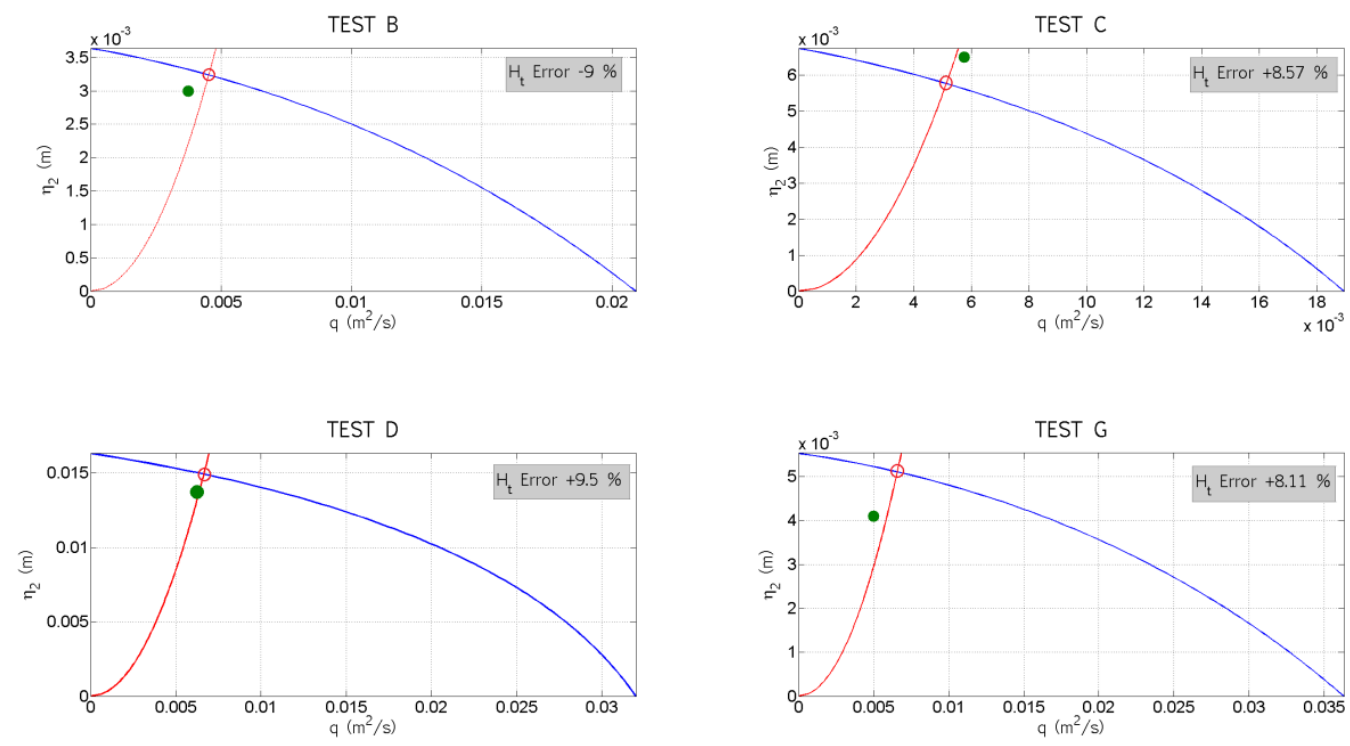

Fig.6 - Operational points for the test cases investigated: measured values (green dot) and analytical values (red circle).

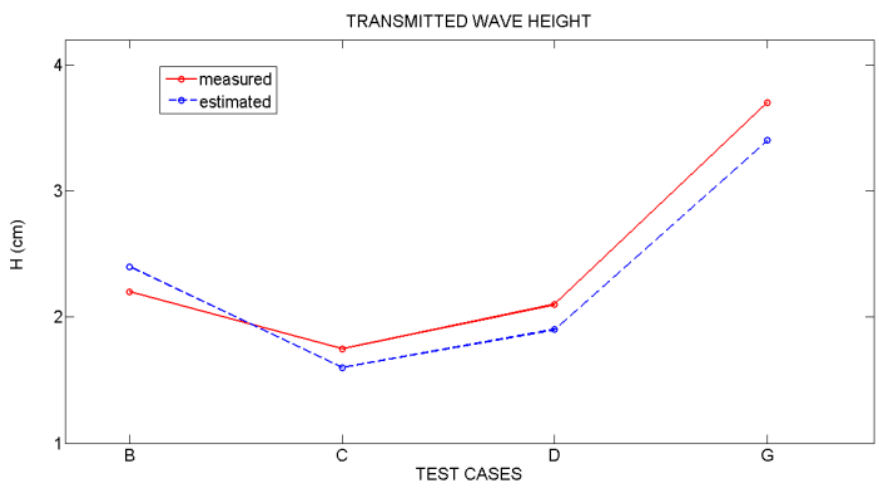

Fig.7 - Height of the transmitted waves for the test cases investigated: measured values (solid line) and values from the empirical formula (dotted line).

\begin{tabular}{|c|c|c|c|c|}
\hline TEST & $B$ & $\mathrm{C}$ & $D$ & $\mathrm{G}$ \\
\hline$\%$ & 9.1 & -8.6 & -9.5 & -8.1 \\
\hline
\end{tabular}

Since the considered laboratory experiments are intended to study the influence of rip channels and submerged sandy bars on the nearshore dynamics, the measurement points are not located close enough to the shore to catch the possible reverse of flow directions. To fill in this gap of information we use the non-hydrostatic free surface numerical model SWASH by Zijlema et al. (2011). The governing equations are the nonlinear shallow water equations including non-hydrostatic pressure. This model can return the three-dimensional structure of the circulation currents by discretizing the water depth in two or more layers. Although a vertical circulation pattern is theoretically possible, here the horizontal twodimensional circulation pattern is supposed to be dominant and the model is run in its depth-integrated form.

In the present work we test the numerical model by comparing wave heights and water levels (Figs 8-11) along two cross-sections with those measured by Haller et al. (2002) in their experiments. Wave set-up is very well predicted for tests B, C and G. Wave breaking related dissipation is slightly overestimated for the transects over the bar. Major differences between measured and predicted values are observed for the transect at the gap of the test D. This can be related to the capability of the 
numerical model to represent the wave blocking by rip currents that did occur for test $\mathrm{D}$ as reported by Haller and Özkan-Haller (2002). The accuracy of the results can be further improved by running the model in its three-dimensional version. For further experimental validation of the SWASH model the reader is referred to Zijlema et al. (2011). The assumption made in the present model of a constant wave set-up $\eta_{2}$ in the cross-shore direction from the inshore toe to the breaking point of the transmitted waves seems to be fairly well supported by the measured values in Figs. 8-11. Measured wave heights indicate that the breaking of waves occurs mainly over and stops just onshore of the structure as assumed in the simple model.
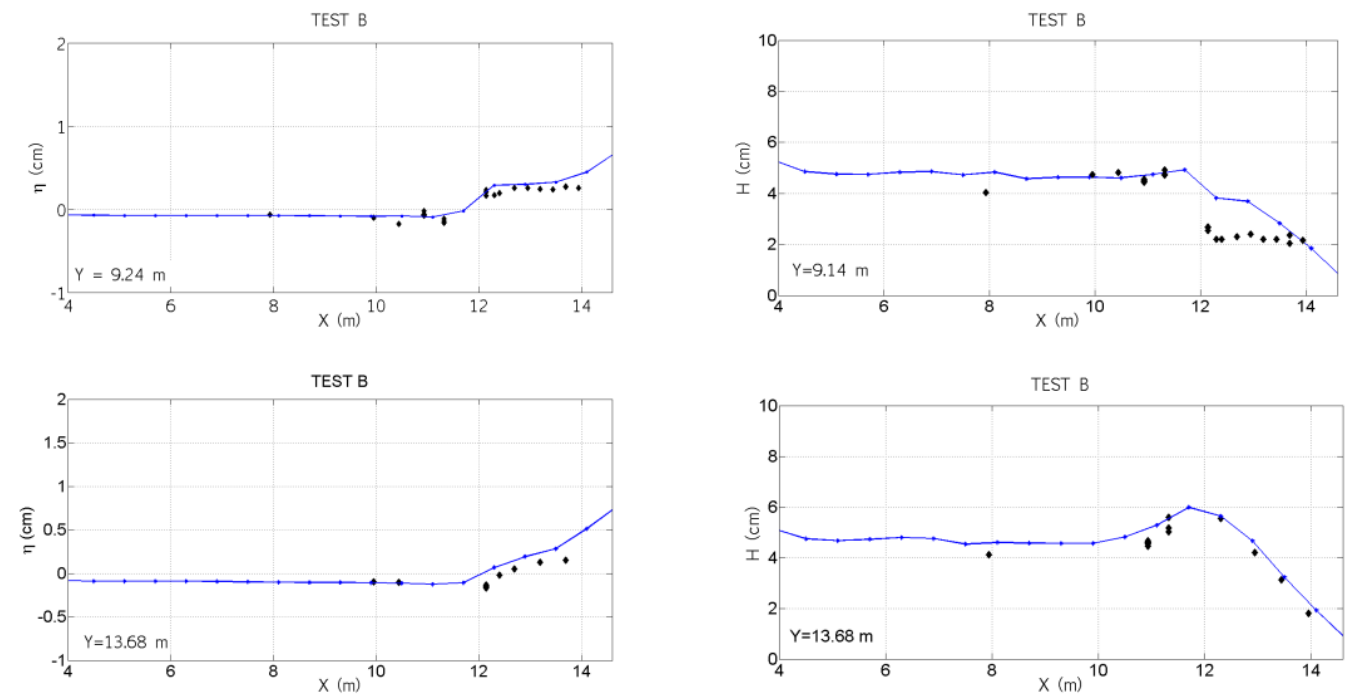

Fig.8 - Experimental (black diamonds) and numerical (solid line) set-up (left) and wave heights (right) for test $B$ for transects in the middle of the barrier (top) and at the gap (bottom).
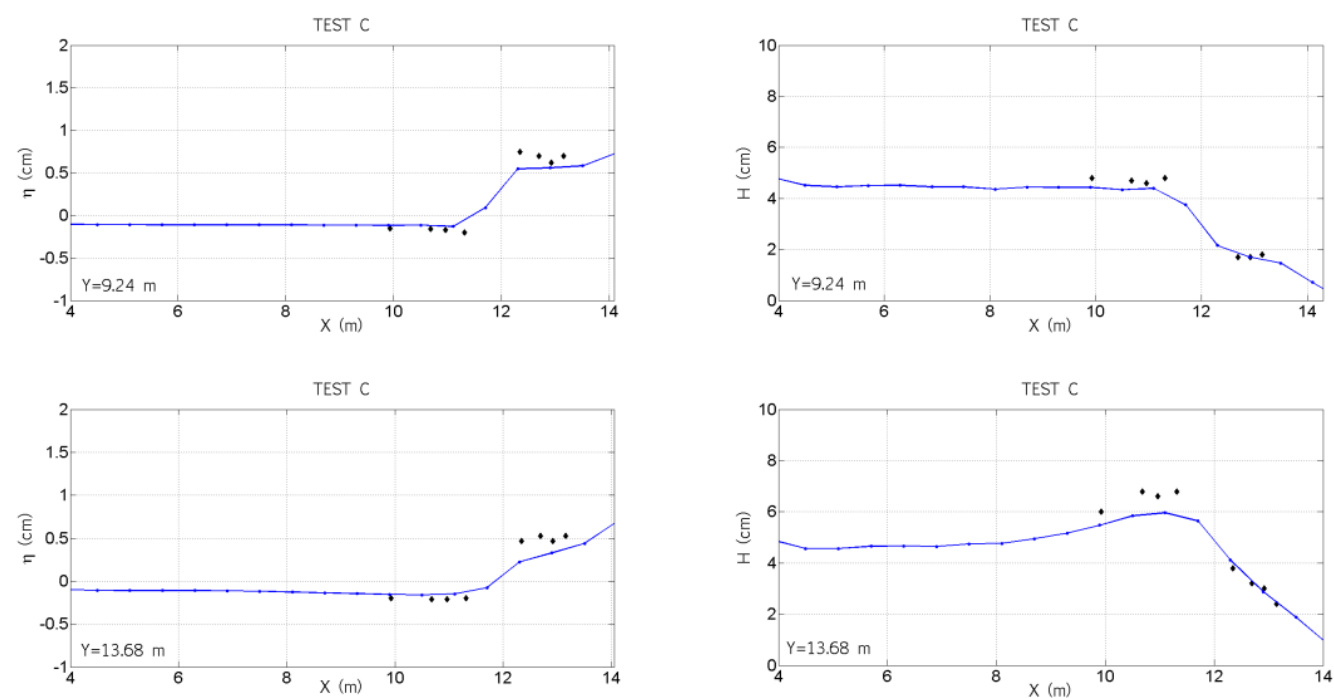

Fig.9 - Experimental (black diamonds) and numerical (solid line) set-up (left) and wave heights (right) for test $\mathrm{C}$ for transects in the middle of the barrier (top) and at the gap (bottom). 

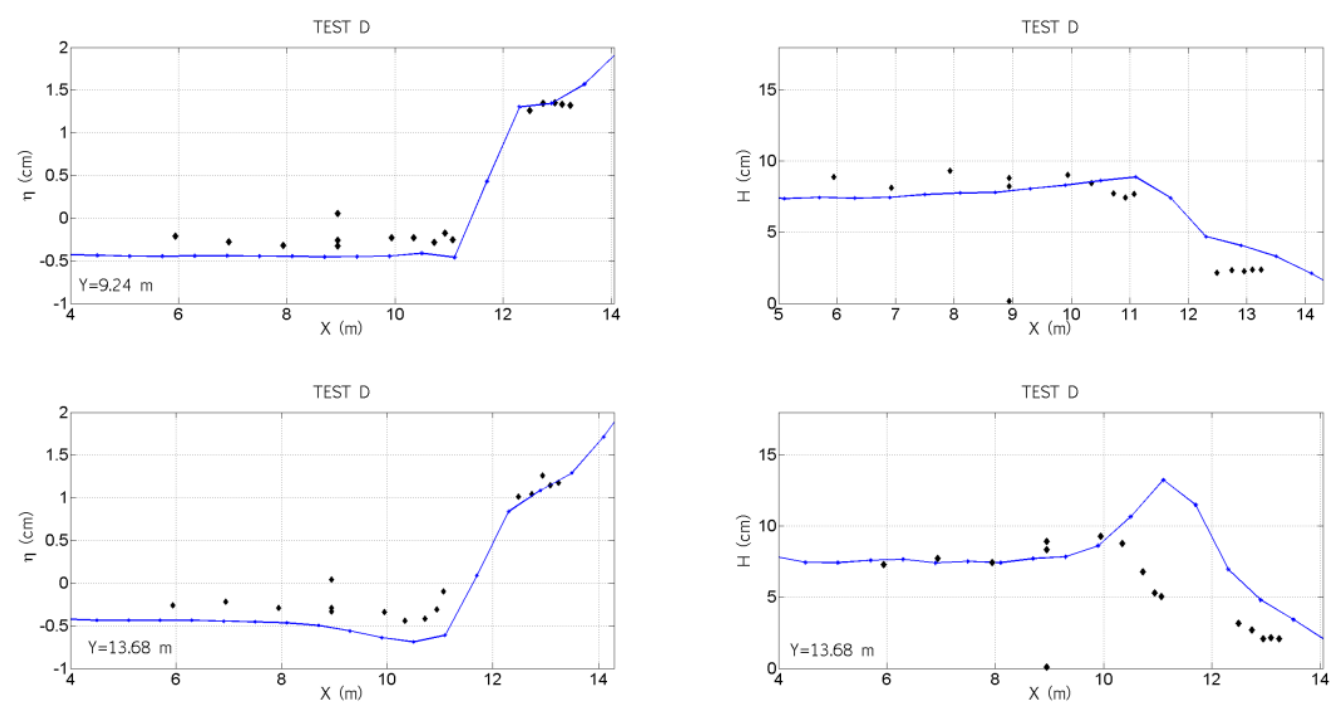

Fig.10 - Experimental (black diamonds) and numerical (solid line) set-up (left) and wave heights (right) for test $D$ for transects in the middle of the barrier (top) and at the gap (bottom).
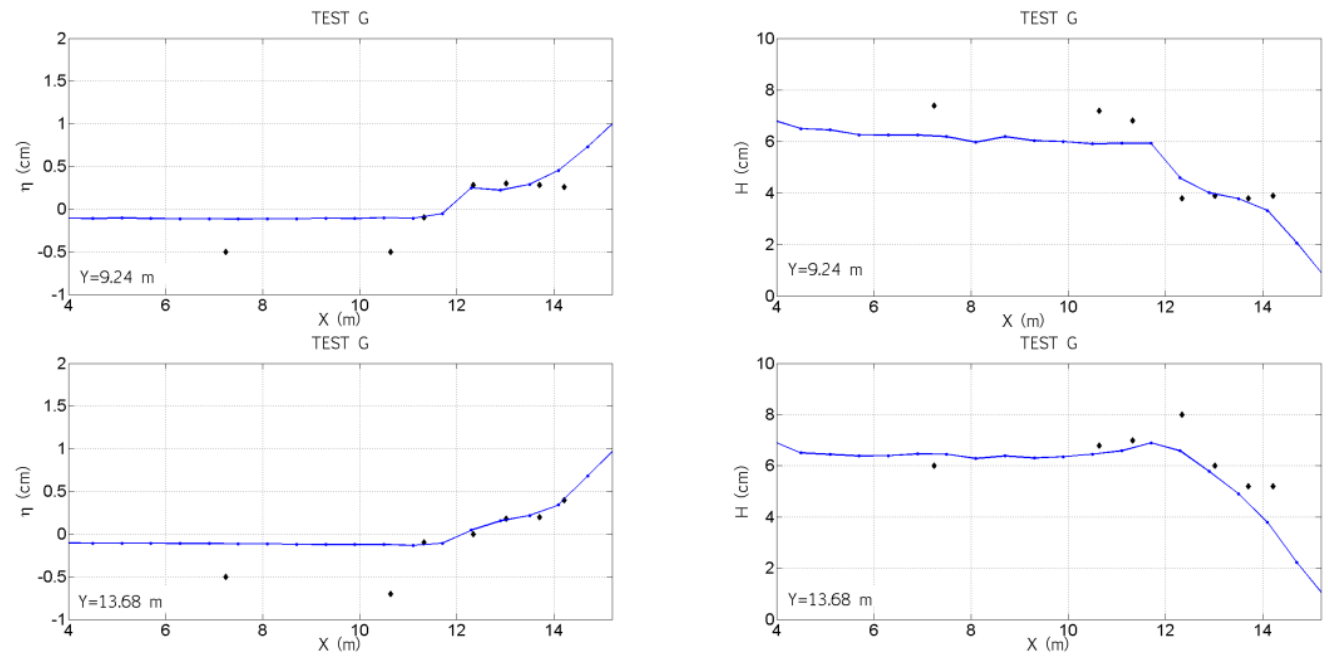

Fig.11- Experimental (black diamonds) and numerical (solid line) set-up (left) and wave heights (right) for test $\mathrm{G}$ for transects in the middle of the barrier (top) and at the gap (bottom).

The simple model returns an accretive mode of response for tests $\mathrm{B}, \mathrm{C}$ and $\mathrm{G}$ and an erosive mode of response for test D (Fig.12). This is in agreement with the time- and depth- averaged currents computed by the numerical model. Figs.13-14 clearly show a 4-cell circulation system for those tests with an accretive analytical response and a 2-cell system for the test with an erosive analytical response. Furthermore, velocity vectors are consistently directed orthogonally to the color map's contour lines indicating wave set-up and set-down. The ratio $r$ is calculated also for the numerical results. In this case the ratio is evaluated using averaged values of the wave setup at the shoreline behind the structure and at the gaps (Tables 3 and 4). The choice to use mean values instead of the values for particular cross-sections removes the sensitivity of the results to the specific cross-section selected.

Applying the criterion to the selected test cases performed by Haller, the errors in $r$ values between analytical and numerical results vary from 3.4 to 6.3\%. As suggested by Haller et al. (2002) the convergent currents close to the shoreline seem to be controlled by the rate of wave penetration through the gaps. For the test cases with greater water depths and smaller rip currents (B and $G)$ the waves dissipation occurring closer to the shore creates greater gradients in radiation stresses and in mean water levels near the shoreline. On the contrary, if the waves approach the coast having already 
dissipated most of their wave energy because of the strong opposing rip currents, the near-shore gradients in radiation stress and mean water levels will be smaller and possibly not sufficient to drive a 4-cell circulation system (test D).

The occurrence of converging currents closer to the shoreline is reported only explicitly for test B of the laboratory experiments, which is the test with the widest grid of measurement points. However, the authors also report that converging currents are "visually observed for all the test cases". This seems to confirm the predictions of the analytical and numerical model for tests B, C and G but to be in contrast with the predictions for test D. On the other hand, considering that the gradients in radiation stress driving the converging currents in test D are essentially zero as reported by Haller et al. (2002) we assume that the specific conditions of wave penetration and opposing rip currents for test $\mathrm{D}$ induce a circulation system that is in a range of transition between completely developed 4-cell and 2-cell circulation patterns. Note further that the strong wave blocking in test D leads to some discrepancies in the gap transect between numerical and analytical model results. Regarding the analytical model the value of the breaker parameter in the gap transect should possibly be taken as dependent on the rip current strength whereas now a constant value for $\gamma_{g}$ is used.

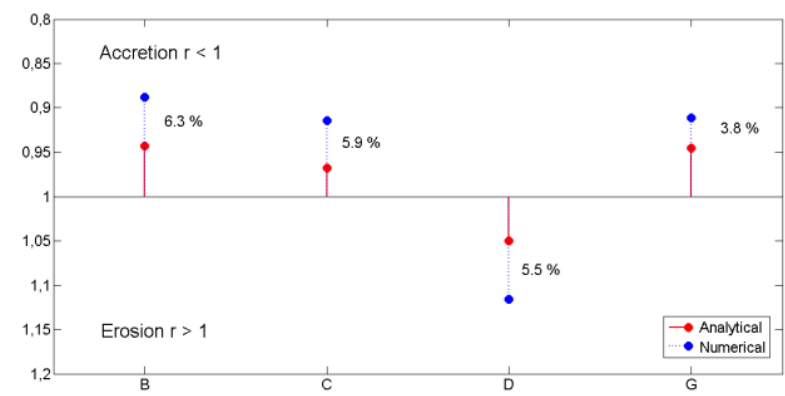

Fig.12- Analytical (red) and numerical (blue) values for the ratio $r$ and percentage difference.

\begin{tabular}{|c|c|c|c|}
\hline & $\begin{array}{c}\eta_{b, a n} \\
{[\mathrm{~m}]}\end{array}$ & $\begin{array}{c}\eta_{b, \text { num }} \\
{[\mathrm{m}]} \\
\end{array}$ & $\begin{array}{c}\text { Percentage } \\
\text { difference }\end{array}$ \\
\hline $\mathrm{B}$ & 0.00875 & 0.0083 & 5.2 \\
\hline C & 0.0091 & 0.0091 & 0.7 \\
\hline D & 0.017 & 0.0154 & 9.4 \\
\hline G & 0.0125 & 0.012 & 4 \\
\hline
\end{tabular}

Table 4. Analytical (an) and numerical (num) values for the wave set-up at the shoreline at the gap.

\begin{tabular}{|c|c|c|c|}
\hline & $\begin{array}{c}\eta_{g, \text { an }} \\
{[\mathrm{m}] 0}\end{array}$ & $\begin{array}{c}\eta_{g, \text { num }} \\
{[\mathrm{m}]}\end{array}$ & $\begin{array}{c}\text { Percentage } \\
\text { difference }\end{array}$ \\
\hline B & 0.0099 & 0.088 & 10.8 \\
\hline C & 0.01 & 0.0094 & 6.3 \\
\hline D & 0.0152 & 0.0146 & 4.2 \\
\hline G & 0.0137 & 0.0127 & 7.5 \\
\hline
\end{tabular}



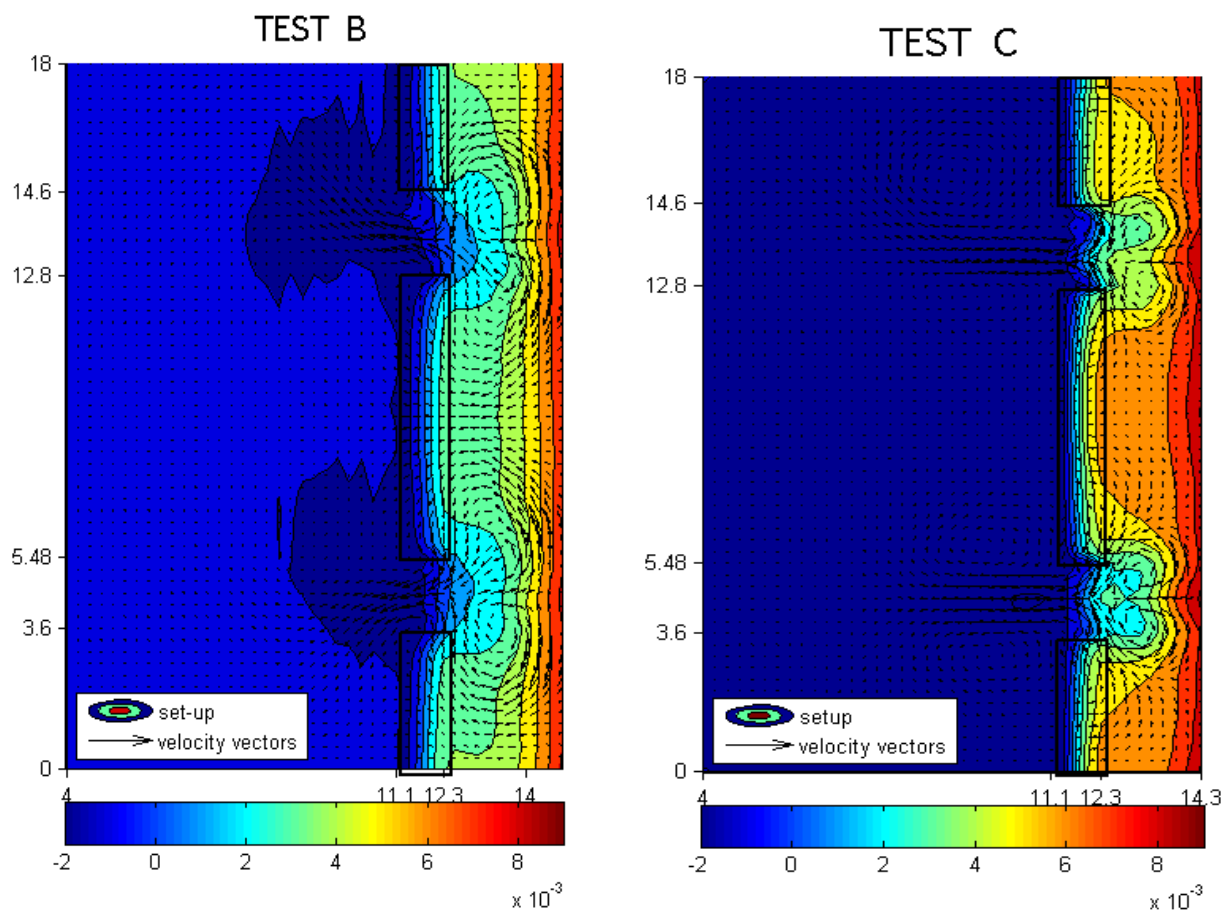

Fig.13 - Plan view of the time-averaged velocity vectors and set-up (color map) for tests $B(r<1)$ and $C(r<1)$.
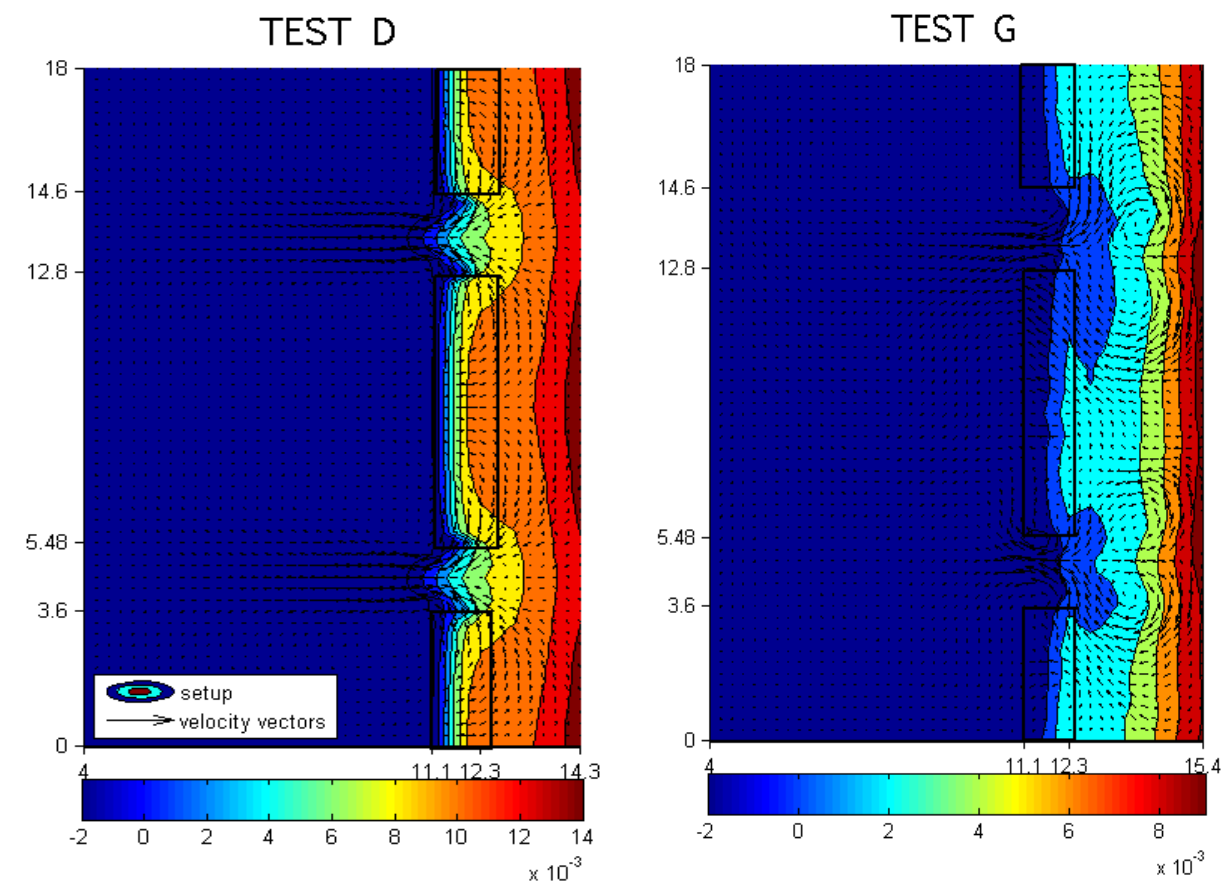

Fig.14 - Plan view of the time-averaged velocity vectors and set-up (colour map) for tests $D(r>1)$ and $G(r<1)$.

\section{CONCLUSIONS}

A criterion to qualitatively investigate the occurrence of either an erosive or accretive circulation pattern in the lee of SBWs is presented. It is based on the assumption that the structure-induced nearshore currents are driven by gradients in mean water levels due to the differential breaking of waves over the barriers and in the gap transect.

The criterion makes use of the model proposed by Bellotti (2007) to evaluate water flow and wave set-up over the structures and assumes a balance between gradients in radiation stress and water level to 
evaluate the wave set-up close to the shoreline. Wave heights in computing the radiation stresses are calculated from linear theory. Although very simple, the presented model and criterion takes into account the wave transformation over the structure, storage of water behind the structures depending on the degree of confinement of the system and breaking of waves on opposing currents.

The model is validated against a non-hydrostatic numerical model (SWASH) that has been successfully verified for a range of experimental data. The validation indicates that the proposed analytical model is capable of correctly identifying the flow directions close to the shoreline, thus providing a rapid first assessment of the potential shoreline response mode for SBW design.

Improvements could be achieved by a better estimation of the values of the transmitted wave heights since the model showed to be very sensitive to this parameter. Further the simple energy equation used as a closure relationship in the Bellotti model can be replaced by a closure relationship that includes some geometrical properties of the system via for instance the length of the return flow path. This would help generalize the model for various environmental and structural parameters.

It was not possible to directly compare the type of circulation system as predicted by the numerical and analytical models to the laboratory tests performed by Haller et al. (2002). These experiments were focussed on the hydrodynamics of rip currents and the locations of the gauges were not chosen such that surface elevations or currents near the shoreline could be measured or the type of circulation could be identified. Nevertheless, the visually observed circulations were in agreement with the model results for three of the four test (B, C and G). For test case D, for which the analytical and numerical model predicted an erosive mode of response, Haller et al. (2002) reported a converging flow close to the shoreline but driven by an essentially zero gradient in radiation stress. We therefore hypothesize that the circulation for test $\mathrm{D}$ is close to a transition from a 4-cell to a fully developed 2-cell pattern.

The model needs to be applied to more laboratory data to test the robustness of the criterion.

\section{REFERENCES}

Bellotti, G. 2004. A simplified model of rip currents systems around discontinuous barriers. Coastal Engineering, 51, $323-335$.

Bellotti, G. 2007. An Improved Analytical Model For Estimating Water level set-up and currents induced by waves over submerged low crested coastal defence structures. Proceedings of the 5th Coastal Structures International Conference, 1, 975-989.

Haller, M.C., R.A. Dalrymple and I.A. Svendsen. 2002. Experimental study of nearshore dynamics on a barred beach with rip channels. Journal of geophysical research, 107(C6).

Haller, M.C. and H.T. Özkan-Haller . 2002. Wave breaking and rip current circulation. Proceedings Of The 28th International Conference on Coastal Engineering, ASCE, 705-711.

Ranasinghe, R. and I.L. Turner. 2006. Shoreline Response to submerged structures: A review. Coastal Engineering 53, 65-79.

Ranasinghe, R., I.L. Turner and G. Symonds . 2006. Shoreline response to multi-functional artificial surfing reefs: A numerical and physical modeling study. Coastal Engineering 53, 589-611.

Ranasinghe, R., M. Larson and J. Savioli. 2010. Shoreline response to a single shore-parallel submerged breakwater. Coastal Engineering 57, 1006-1017.

Van der Meer, J.W., R. Briganti, B. Zanuttigh and B. Wang. 2005 Wave transmission and reflection at low crested structures: Design formulae, oblique wave attack and spectral change. Coastal Engineering, 52915-929.

Vicinanza, D., I. Caceres, M. Buccino , X. Gironella and M. Calabrese. 2009. Wave disturbance behind-low crested structures: Diffraction and overtopping effects. Coastal Engineering 56, 11731185.

Zanuttigh, B., L. Martinelli and A. Lambert. 2008. Wave overtopping and piling-up at permeable low crested structures. Coastal Engineering, 55, 484- 498.

Zijlema, M., G. Stelling and P. Smit. 2011. SWASH: An operational public domain code for simulating wave fields and rapidly varied flows in coastal waters. Coastal Engineering, 58, 992-1012. 